Connection MiesSANAA.

On intervention by Sanaa in

Barcelona Pavilion of Mies

PALABRAS CLAVE I MIES VAN DER ROHE I SANAA | PABELLÓN DE BARCELONA | ESPACIO | SIMETRÍA

KEYWORDS | MIES VAN DER ROHE | SANAA | BARCELONA PAVILON I MACE ISYMATER

\section{| RESUMEN |}

Al margen del juego de palabras del título, la influencia de Mies en SANAA es evidente tanto en el reconocimiento de los propios arquitectos como por los últimos estudios que los vinculan. Ahondado en esta "conexión", un ejemplo significativo es la intervención que Kazuyo Sejima + Ryue Nishizawa realizaron en el Pabellón de Barcelona a finales de año 20ll. Introdujeron un muro curvo transparente de material acrílico que "casi" sin alterar el conjunto envolvía el muro de ónice de la "sala del trono". Un gesto primario, la materialización de un recorrido, una espiral "topológica" que señalaba un centro que paradójicamente se disuelve a causa de los múltiples reflejos que la intervención provoca. Un manifiesto iniciático que revela las intenciones futuras de los auténticos herederos de Mies. La intervención en el Pabellón alemán se convierte así en una ofrenda sobre el "altar disuelto" de lo que representa el templo de la arquitectura moderna.

\section{| ABSTRACT |}

Outside the pun in the title, the influence of Mies in SANAA is evident both in the recognition of the architects themselves and in the recent studies that link them. Delving into this "connection", a significant example is the intervention Kazuyo Sejima + Ryue Nishizawa made in the Barcelona Pavilion at the end of 20ll.They introduced a transparent curved wall made of acrylic material that "almost" without altering the set enveloped the onyx wall of the "throne room". A primary gesture; the materialization of a tour, a "topological" spiral pointing to a center which paradoxically dissolves due to multiple reflections that the intervention provokes. An initiation show that reve is the future intentions of the true heirs of Mat revel The intervention in the Cerman pavilon thus Mies. The intervention in the German pavilion thus becomes an offering on the "dissolved altar" of what represents the temple of modern architecture.

\title{
Conexión MiesSANAA. Sobre la intervención de Sanaa en el Pabellón de Barcelona de Mies
}

\author{
FRANCISCO MUÑOZ CARABIAS*·Universidad Alfonso X El Sabio, España·fmunocar@uax.es
}

Fecha de recepción 09/septiembre/2015 · Fecha de aceptación 13/diciembre/2015

\section{¿ARQUITECTOS MIESIANOS O UNA ARQUITECTURA MIESSANAA?}

"Conexión MiesSANAA" es un juego de tres palabras contenida en dos. "Conexión", como relación, en este caso, entre dos arquitecturas separadas en el tiempo y en el espacio ${ }^{[1]}$ y "MiesSANAA", que surge de unir Mies + SANAA, que alude directamente al parecido fonético con el término "miesiano", (Rowe, 1999 , p. 22) en referencia a los supuestos herederos del maestro alemán. Oficialmente, esta condición recayó en sus discípulos americanos y en las generaciones de arquitectos que se formaron bajo su dirección en la Escuela de Arquitectura del Illinois Institute of Technology (IIT) de Chicago, siguiendo un plan de estudios diseñado por el propio Mies van der Rohe. Pero su influencia excede con diferencia estos límites geográficos y temporales ampliando su extensión a todo el siglo XX ${ }^{[2]}$. De hecho, podríamos estudiar la arquitectura de los últimos cincuenta años en clave de afinidad o rechazo hacia lo que la figura de Mies representó. Sobre estos sentimientos extremos, haciendo homenaje a los términos opuestos que tanto llegó a emplear, el legado dejado ha podido ser entendido desde múltiples maneras, algunas antagónicas. Los "miesianos" del IIT, que desarrollaron su actividad entre la década de los cuarenta a los

* Profesor de Proyectos Arquitectónicos 3 en la Universidad Alfonso X El Sabio de Madrid, España. Coordinador de esta asignatura y de Proyecto Fin de Carrera (PFC) en la misma universidad. Coordinador del Máster de Proyectos Arquitectónicos Avanzados, Universidad Alfonso X El Sabio de Madrid. Miembro del grupo de investigación "Geometrías de la Arquitectura Contemporánea" de la Escuela Técnica Superior de Madrid (ETSAM) de la Universidad Politécnica de Madrid (UPM), España. Tesis doctoral titulada "La paradoja de Mies. Las simetrías invisibles a través del Pabellón de Barcelona" dentro del Doctorado en Teoría y Práctica del Proyecto del Departamento de Proyectos Arquitectónicos en la ETSAM, UPM. Arquitecto fundador del estudio taller TRAZAARQUITECTURA (www.traza-arquitectura.com). Autor de diversos proyectos, algunos de ellos fruto de concursos ganados.

[1] En topología, es un invariante que se usa para distinguir espacios topológicos diferentes: conexos e inconexos. Parece importante mencionar esta relación con la topología. Juan Antonio Cortes en su artículo titulado "Topología Arquitectónica" comenta lo siguiente: "la obra de Sejima y de Nishizawa puede ser considerada un manual de topología arquitectónica". En cuanto a la palabra "topología", como Lacan la usa repetidamente en el sentido de relaciones que no son métricas. "Bueno, esto es topológico y no geométrico" queriendo decir con eso que no importan las medidas, importa la relación entre elementos, y cómo esos se relacionan.

[2] Las palabras que pronunció Koolhaas, a propósito de la propuesta para un nuevo centro de estudiantes en el Campus IIT en el año 2000, denotan el intenso de debate que siempre ha existido sobre su figura: "Yo no respeto a Mies, amo a Mies./He estudiado a Mies, excavado a Mies, reensamblado a Mies. He incluso limpiado a Mies./ Como no reverencio a Mies, estoy peleado con sus admiradores... Mies necesita ser protegido de sus defensores". (Koolhaas, 2004, p. 44) 
1. Casa Resor. Modelo. Hedrich Bleassing, HILBERSEIMER, Ludwig; Mies van der Rohe, Turín: Città Studi Edizioni, 1993. $1^{a}$ Edición, 1956. 165 p. ISBN: 978-88-251-0076-1, p. 74. № de fotografía: 50. Modello.

2. Planta. Museo para una pequeña ciudad. A.Monistiroli, HILBERSEIMER, Ludwig; Mies van der Rohe, Turín: Città Studi Edizioni, 1993. $1^{a}$ Edición, 1956. 165 p. ISBN: 978-88-251-00761, p. 146. № de fotografía: 149. Planta. Museo per una piccola città.
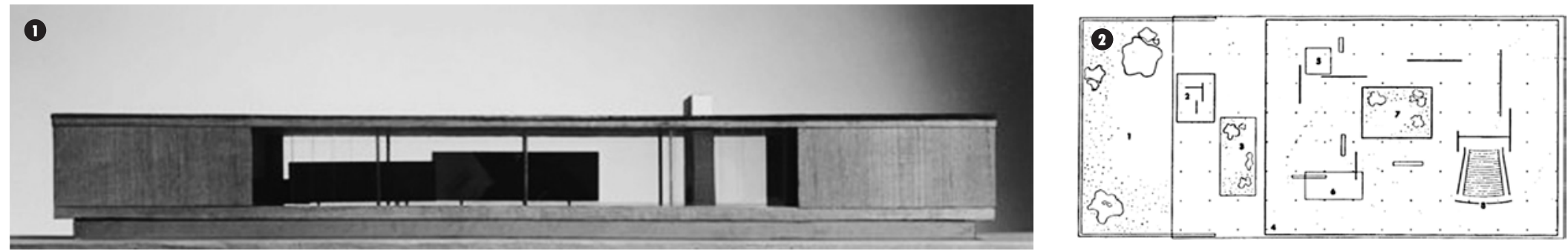

sesenta en los EE.UU., pasaron de representar la ortodoxia de la modernidad a ser calificados, a finales de este período, como una arquitectura monótona en el seguimiento acrítico de una esencialidad malentendida. "Less is bored" (menos es aburrido) fue la sentencia de Robert Venturi ante la proliferación de una infinidad de cajas ${ }^{[3]}$ resueltas en una rigurosa ortogonalidad que podían servir tanto para una vivienda, como para una escuela o un teatro. Una estética depurada de pieles de vidrio, estructura de acero y cubierta plana que llegó a convertirse en un repertorio estilístico más al uso. ¿̇Qué pudo ocurrir? Quizás del "less is more" (menos es más) que caracterizó la idea primigenia de Mies se pudo pasar por desidia y cansancio al "less is less" (menos es menos) de sus herederos. Lo primero fue, y es, una paradoja productiva, lo segundo, sin embargo, es, y será siempre, una identidad estéril. Lo primero representa un principio de equivalencia de contrarios que revela un grado de complejidad dentro de la similitud planteada que se aleja de la correspondencia literal del segundo. Es, como se verá más adelante, la diferencia entre la construcción de un orden basado en una definición ampliada de la simetría o la visión reductiva que se ha tenido siempre de esta. Es esa "conexión MiesSANAA" planteada en el título o la "mimesis miesiana" mantenida por los segundos.
Pero yendo al principio del asunto, los dos primeros proyectos que Mies realiza en América al abandonar Alemania, son la casa Resor (1937) y el Museo para una Pequeña Ciudad (1942). Ambos deberían continuar lo culminado en Europa con el Pabellón Alemán para la Exposición Universal de Barcelona de 1929 y la casa Tugendhat en Brno pero manifiestan, sin embargo, las complejas contradicciones del pensamiento miesiano (esta vez referido al propio Mies). Un pensamiento, que en palabras de Peter Smithson "discurre por cauces profundos y no fácilmente accesible. Se sospecha que ni para él mismo" (Navarro Baldeweg, 1999, p. 77) como muestran estos dos ejemplos. Así, la casa Resor (Figura 1) expresa, en la imagen de la maqueta de lo que fue la última versión del proyecto que finalmente no llegó a realizarse, una evidente simetría bilateral en su exterior.

Como contrapunto a esa simetría objetual, el Museo para una Ciudad Pequeña (Figura 2) fruto del desarrollo del proyecto fin de carrera de uno de sus alumnos, George Danforth, (Schulze, 1986, p. 230), pone el énfasis en la simetría modular de una retícula neutra de pilares cruciformes como prueba de una isotropía espacial sin direcciones privilegiadas característico de la planta libre moderna ${ }^{[4]}$. Podría decirse, por comparación, que este proyecto está arquitectónicamente "más evolucionado" que la casa Resor. Pero las salas hipóstilas son tan antiguas en la arquitectura como aquellas otras nacidas de simetrías con un eje principal. De hecho ambos órdenes llegan a combinarse en la mayoría de los casos conocidos, estableciendo sus diferencias en las jerarquías planteadas entre sus elementos. ¿Dónde está por tanto la novedad? Para ello será necesario comparar los interiores de los dos proyectos y comprobar la asombrosa similitud de ambos (FIguras 3 y 4). Una serie de muros, de diversas "texturas", representando a su vez diferentes materiales fuera de escala, flotan en este "continuum espaciotemporal" limitado por un plano superior e inferior que en las perspectivas se confunden con el fondo blanco. Incluso el propio paisaje exterior es tratado como otro material más. $\bigcirc$ más bien, como plano de imagen (in)material del mismo. Un vacío sin referencias dimensionales que es el acercamiento más certero que ha habido a ese espacio concebido por la física de la relatividad de Einstein de tomar como premisa de indeterminación su naturaleza continua y homogénea. Es la "conexión", sesenta años después, entre lo intuido por Mies y lo proyectado por SANAA en el Park Café (1998) de Osaka (FIGURA 5) o el concurso para el Museo de la Mercedes Benz en Stuttgart (2002).

[3] Colin Rowe en el ensayo "Neoclasicismo y arquitectura moderna I" ya advierte de esta herencia que la equipara al neopalladiano.

[4] La planta libre concebida dentro del Movimiento Moderno, es dinamizada a través del juego de volúmenes y tabiques verticales de modo similar a las obras de Le Corbusier y a las experiencias de los stands expositivos realizados para la industria alemana que Mies hizo junto a Lily Reich. 
Los miesianos de Chicago, sin embargo, no pudieron, porque quizás no supieron, ver lo que estaba detrás de esta enigmática arquitectura que sí ha sido descifrada ahora por el estudio japonés. Una arquitectura supuestamente simple, desnuda, mínima, de plantas, incluso simétricas en el sentido clásico, pero desconcertantes y cambiantes en su experiencia espacial real. Tuvo que darse un cierto grado de madurez para que se llegase a producir este cambio de visión. Una madurez responsable del propio redescubrimiento del Pabellón de Barcelona, prácticamente ignorado durante su corta existencia material en 1929 y que fue cobrando importancia a lo largo de los años a través de unas pocas imágenes en blanco y negro. La culminación de este camino como mito de la arquitectura moderna lo representó su reconstrucción del año 1986 por parte de un grupo de arquitectos catalanes, Solà-Morales, Cirici y Ramos, que entendieron esta intervención más allá de la mera réplica, y que en términos simétricos, sería tanto como decir que pasaron de la identidad a la equivalencia.

La influencia de Mies en la arquitectura de SANAA es, por tanto, clave para establecer esta conexión. Algo, por otra parte, conocido por los propios actores, Kazuyo Sejima y Ryue Nishizawa, pero también, necesario para su superación. "SANAA analiza, interpreta, modifica, destruye y reconstruye el sistema proyectual de Mies" (Jaraiz, 2013, p. 29) porque se entiende que en él está la base de una nueva arquitectura. Más en concreto, hay la certeza de que la década de los veinte, los cinco proyectos "heroicos" de Mies: Los dos rascacielos de vidrio, el edificio de oficinas de hormigón y las casas de campo de ladrillo y hormigón, junto con los ya mencionados, el Pabellón de Barcelona y la casa Tugendhat, fueron la culminación, y no el inicio, de un proceso que hizo posible la expresión de unos ideales de modernidad huérfanos de una imagen icónica que los contuviera. Muchos proyectos de SANAA comparten este papel clave en la actualidad desde esta legitimidad que los alberga. Evidentemente este hilo "conector" entre Mies y SANAA va más allá de su posición en el
3. Perspectiva interior de la Casa Resor. Código: 0121641 . Museum of Modern Art (MoMA) - New York. Título: Resor House, project (Jackson Hole, Wyoming): Perspective of living room through south glass wall. 1937-1941 (unbuilt). Copyright: (C) Museo de Arte Moderno. Departamento de Servicios de Imagen del MoMA, 11 West 53 Street, Nueva York, Nueva York, 10019, EE.UU.

4. Perspectiva interior del Museo para pequeña ciudad. Código: 0123662. Museum of Modern Art (MoMA) - New York Título: Museum for a Small City, 1942. Interior perspective. Copyright: (c) Museo de Arte Moderno. Departamento de Servicios de Imagen del MoMA, 11 West 53 Street, Nueva York, Nueva York, 10019, EE.UU.

5. Cristal y espejos. Interior del Park Café en Koga. SANAA. Autor: Louise Gronlund.
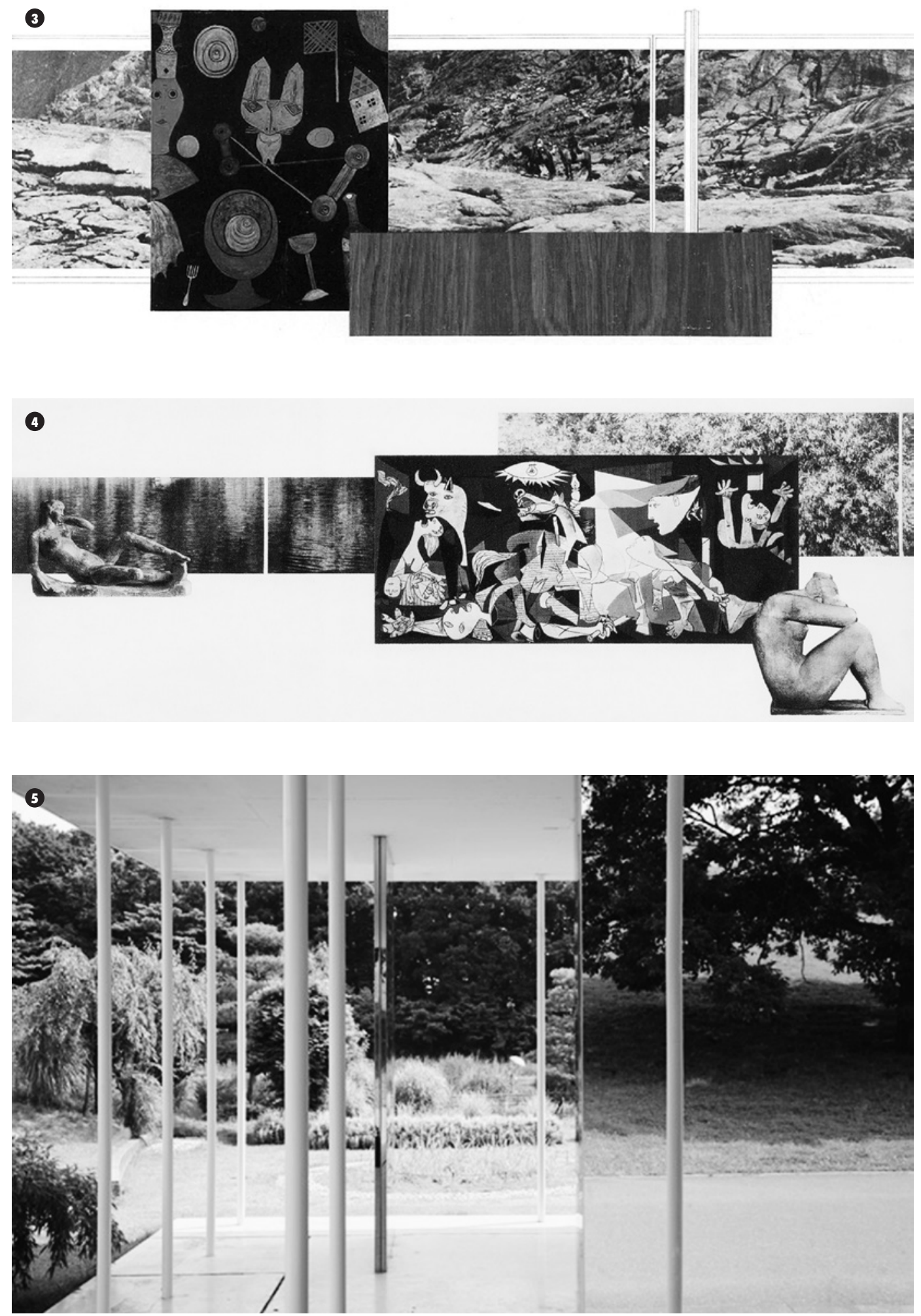
panorama arquitectónico, en este momento, y sí en cambio, tiene más que ver con expresar y establecer los elementos esenciales que constituyen todavía el proyecto moderno. Uno de los más destacados es la idea de espacio y las geometrías asociadas a él, que se dieron en se momento, superando el marco geométrico euclideo habitual, y que han vuelto a hacerse presentes al darse las condiciones evolutivas arquitectónicas que lo han posibilitado.

\section{LAS SIMETRÍAS CONTINUAS. LAS SIMETRÍAS DEL ESPACIO}

El desarrollo matemático del siglo XIX permitió que el "espacio" fuese tratado como un ente independiente del espacio físico, con sus propias reglas, cuando hasta el momento había sido indistinto en su apreciación de la realidad que habitamos. La creación de los espacios abstractos, a comienzos del siglo XX, culmina un proceso de emancipación que destierra el espacio concebido como contenedor por otra "entidad abstracta que consiste en un conjunto cualquiera de objetos y una red de relaciones entre ellos" (Corrales, 2002, p. 23). Precisamente el dominio de la abstracción ${ }^{[5]}$ como sistema de representación en el arte moderno encontró un punto de encuentro en el proceso que hizo que se invirtieran los términos y fuese el espacio matemático el que alcanzase una legitimidad que antes la había tenido el físico-real. Espacio, que en el Movimiento Moderno, siguió siendo el gran vacío newtoniano isótropo, cúbico e intemporal conocido, pero que en obras como el Pabellón, dio un salto cualitativo hacia esta nueva concepción del mismo. "Solo contiene espacio. Espacio de una composición geométrica, intangible, inmaterial" ${ }^{[6]}$ dirá Rubio i Tudurì en el año 1929 ante la conmoción que le causó ver en directo el Pabellón de Barcelona y lo que supuso para la arquitectura moderna entender el proyecto desde el orden de las simetrías del espacio y no de la materia. Su estructura interna, reconocida ya desde el Renacimiento, se reveló más nítidamente en esos momentos precisamente en geometrías donde hubo un incremento en la invariancia, y por lo tanto de la simetría asociada al marco de referencia que tenemos de mirar y reconocer esta realidad ${ }^{[7]}$. En palabras de Juan Navarro recogidas en su texto "Movimiento ante el ojo, movimiento del ojo", "es esta simetría la evidencia más fuerte de una cualidad abstracta en el espacio que se destaca por encima de su apariencia figurativa" ${ }^{[8]}$. Un concepto ampliado que permite entender que la isotropía espacial no es otra cosa que un reconocimiento explícito y expreso de la estructura homogénea del universo cuyas cualidades fueron definidas en las simetrías continuas postuladas en el Teorema de Noether ${ }^{[9]}$ (Lederman \& Hill, 2006).
Sobre el conocimiento por parte de Mies del mismo no podemos pasar del plano de la conjetura, como por otra parte fue habitual en una figura que dejó tan solo catorce escritos entre artículos y discursos, y ningún libro publicado. Si su pensamiento se ha podido recomponer a través de sus lecturas y de su biblioteca privada ${ }^{[10]}$, desde esta premisa, hay razones para pensar de que al menos tuvo las bases para ello cuando no la intuición de esta nueva realidad. Un ejemplo de esto, es el siguiente comentario encontrado en su cuaderno de notas: "Los últimos veinte años han comportado una subversión del pensamiento científico, cuyo significado para todo lo venidero aún no puede ponderarse" [1]]. En una conferencia pronunciada en la Biblioteca Nacional de Berlín en 1929, al insistir en el papel decisivo de la técnica, pronuncia estas palabras sobre una imagen de las calles de Nueva York: "... transforma la materia en energía" [12] que pone en evidencia un lenguaje común, a finales de los años veinte, de las teorías sobre la Relatividad General de Einstein y el principio de equivalencia que rige el universo en la conservación de la energía y de el Teorema de Noether que lo desarrolla. De ahí el interés por la ciencia ${ }^{[13]}$ y en especial por la astrofísica ${ }^{[14]}$, que le acompañará hasta el final de su vida. Una veintena de libros de su propiedad, versan sobre esta física moderna, de los cuales, cuatro al menos, recogen las teorías indicadas. De

[5] No como proceso de abstracción, sino como representación de una espacio abstracto desligado del mundo de la naturaleza. Capi Corrales hace la comparación entre un cuadro de Mondrian y Cezanne (Proceso de abstracción) y Kandinsky (representación de un espacio abstracto).

[6] En 1929 se publicó en Cahiers d'Art (1929: 175) la descripción del Pabellón escrita por Rubio i Tuduri.

[7] "La geometría siempre ha partido de la observación de la realidad. Diferentes realidades han motivado diferentes modelizaciones geométricas" C. Alsina y E. Trillas (1983) Lecciones de Algrebra y Geometria. Curso para estudiantes de Arquitectura. Barcelona: Gustavo Gili, p. 12.

[8] Navarro Baldeweg, La habitación vacante (p. 176). Gerona: Pre-textos. "Movimiento ante el ojo, movimiento del ojo", p. 21.

[9] La simetría abarca todas las escalas de la realidad "desde el mundo íntimo de la estructura de la materia hasta el cosmos" (Lederman \& Hill, 2006) y las leyes físicas fundamentales que lo rigen se basan en este concepto. A través del teorema de Noether dice que "por cada simetría continua de las leyes físicas existe la correspondiente ley de conservación", se llega a la conexión entre el corpus matemático de la simetría y el comportamiento dinámico de los sistemas físicos. El teorema pone de relieve que la simetría es el principio subyacente más trascendental de la naturaleza pero a la vez, adjetiva la simetría con el término continuo.

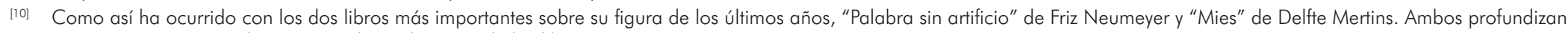
en su pensamiento mediante un análisis exhaustivo de los libros que poseía.

[11] Neumeyer, Mies van der Rohe: la palabra sin artificio. Reflexiones sobre arquitectura 1922-1968. p. 418. Mies van der Rohe; Cuaderno de notas, hoja 7. 1928.

[12] Ibid. p.455. Mies van der Rohe: Conferencia "Los requisitos de la creatividad arquitectónica" 1928.

[13] Su hija Georgia dice que todo empezó al encontrar un ejemplar de la revista "El futuro" en la mesa de dibujo que le asignaron en el primer estudio de arquitectura que trabajó (Schneider). Ver Schulze, Mies van der Rohe. Una autobiografía crítica. p. 18.

[14] David Spaeth, Mies van der Rohe (Barcelona: Gustavo Gili, 1986). p. 22. 
Niels Bohr, LA FÍSICA ATÓMICA Y CONOCIMIENTO HUMANO Y de Schrodinger, MENTE Y MATERIA Y LA CIENCIA Y EL HUMANISMO: LA FÍSICA EN NUESTRO TIEMPO. Sin descartar un libro clave entre sus favoritos, Simetría de Hermann Weyl, del que fue amigo y profundo admirador de Emmy Noether.

Los criterios configuradores de la forma permanecieron, por tanto, para Mies indeterminados en gran medida. La posición antiestética que manifestaba durante el período de las vanguardias, bloqueaba cualquier materialización formal coherente con su discurso. Solo desde la configuración "negativa" del espacio como "no-materia", podía resolverse esta "paradoja" de naturaleza no-formal que a la postre significó la posibilidad de una construcción efectiva del espacio independiente de su delimitación. Esto nos conecta con la arquitectura japonesa contemporánea y la enorme influencia que Mies ha tenido en estos últimos años desde "los límites difusos" de Toyo Ito hasta la nueva generación de arquitectos, donde destacan, a parte de Kazuyo Sejima + Ryue Nishizawa (SANAA), Junya Ishigami. Es curioso este camino de ida y vuelta que se ha dado entre Oriente y Occidente en el caso de la arquitectura. Si la influencia inicial de Oriente en la arquitectura de Movimiento Moderno se evidencia en aspectos como la concepción espacial y la esencialidad del tratamiento de la materia, a raíz, entre otras experiencias, de la exposición que Wright realizó de su obra en Berlín en 1910; en el caso de la arquitectura japonesa, el reconocimiento de esta influencia occidental, es sobre todo, obra de Mies (y en parte también del propio Le Corbusier). No obstante, en todo este proceso, y al margen de las propias referencias proyectuales, el valor icónico se sigue manteniendo con extraordinaria fuerza pregnante. Si el portfolio Wasmuth (catálogo recopilatorio de los dibujos de la
6. Vista del Pabellón de Barcelona desde el estanque mayor. 1986. Autor: Francesc Català-Roca @ Fundació Mies van der Rohe y Fundació Francesc Català-Roca.

7. Vista del Pabellón Serpentine Gallery. 2009. Kazuyo Sejima \& Ryue Nishizawa (SANAA). Fuente: The Architecture review. Fotografía: Ludwig Abache.

8. Pabellón del vidrio del "Toledo Museum of Art". Toledo (Ohio) (EE. UU.). 2013. Kazuyo Sejima \& Ryue Nishizawa (SANAA). Fuente: Tectonicablog. Fotografía: Trevor.patt y Tim Brown Architecture.
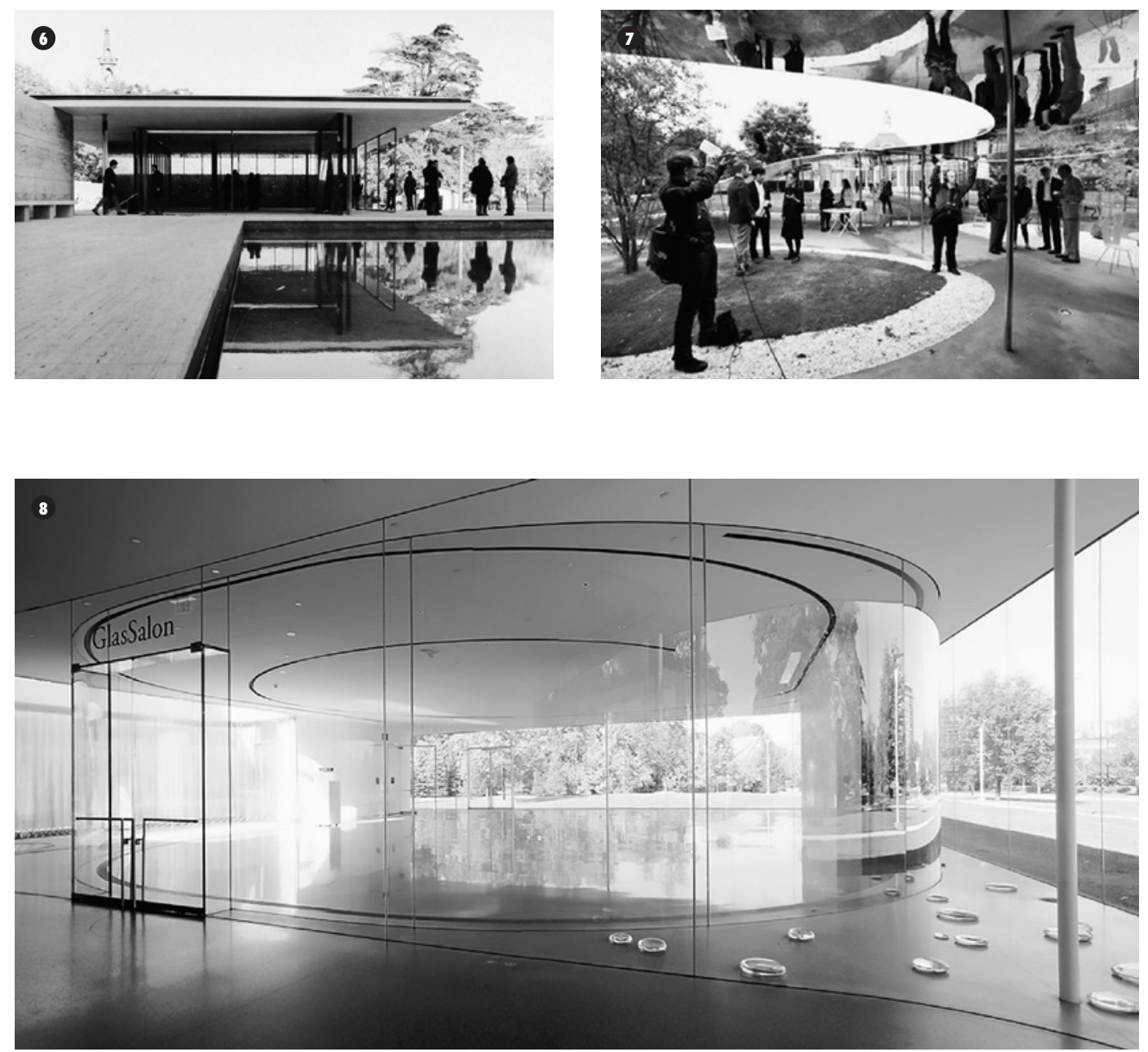

exposición de Wright) que conocieron, entre otros, Le Corbusier, Gropius y Mies en el estudio de Behrens, influyó de manera notoria en los medios de expresión de la arquitectura ${ }^{[15]}$; en el caso de SANAA, el uso de las imágenes míticas de los proyectos de Mies de los años veinte, es la primera aproximación a su obra; a la cual, trata de imitar, por analogía visual directa.
De todos modos, si nos adentramos en otras "conexiones" [16] SANAA radicaliza lo planteado por Mies en lo referente a la simetría como un principio de equivalencia entre elementos desde la invariancia. Esta definición incorpora lo recogido por Lederman como "expresión de igualdad", así como lo planteado por Weyl como "invariancia de una configuración bajo

[15] A través de unas imágenes "alteradas" que el propio Wright realizó en un grafismo contrastado de luces y sombras, en blanco y negro procedentes de la estética japonesa.

[16] En los códigos formales de su lenguaje, SANAA asume en mayor medida el corpus corbusiano que el miesiano al menos en su primera época: uso del blanco "purista" en casi todos los cerramientos e interiores, volúmenes nítidos y de geometría rotunda. Niega toda referencia al material que en Mies se realiza constantemente. La relación con Mies es más intelectual y se basa más en aspectos ligados a la idea de espacio. 
un grupo de automorfismos". Precisamente para Weyl, el espacio vacío tiene un alto grado de simetría. "Cada punto es igual que los otros" (Weyl, p. 16) y es este vacío el que se pretende recobrar para la arquitectura.

El espacio horizontal presente en el Pabellón de Barcelona (FIGURA 6) es un eco de este vacío que se vuelve a repetir en los proyectos de SANAA (como en la Serpentine Gallery de 2009) (FIGURA 7) mediante un eje de simetría también horizontal. Esta simetría invertida, donde "el mundo (es) vuelto al revés" (Evans, 2005, p. 274), hace prevalecer la extensión en las dimensiones " $\mathrm{x}$ " $\mathrm{e}$ " $\mathrm{y}$ ", consiguiendo anular la coordenada " $z$ ", y por tanto, la gravedad a través de un mecanismo de equivalencia entre el suelo y el techo. La planta libre generada se desarrolla entre estos dos planos frontera que establecen una discontinuidad en el continuum que seccionan. "Podrá variar en algunas obras el número de planos horizontales, pero siempre persistirá esta condición: el sinfín de un espacio ilimitado que queda cobijado en el ambiguo confín ${ }^{[17]}$ que forman los dos planos de naturaleza distinta" (Martínez Santamaría, p. 67). En SANAA hay un significado amplificado de este esquema mediante un incremento de su isotropía. La estructura en Mies se sigue ciñendo a la retícula cartesiana en los nudos que todavía poseen una condición diferente al resto. "Los puntos, aristas y planos (de la retícula tridimensional ortogonal homogénea) adquieren un carácter privilegiado: evidencian un orden reticular objetivo..." (Ynzenga Acha, 2013, p. 101).

La propuesta de SANAA es disolver esta última jerarquía espacial mediante la multiplicación en infinitos pilares reducidos, llevados a la mínima expresión, disolviéndose con el resto de elementos del edificio. La "eliminación" de la estructura, uno de los sueños que Mies trató de alcanzar hasta el final, lleva a profundizar en la propia desaparición de la arquitectura. Del "beinahe nichts" (casi nada) al "nichts" (nada) para ser ocupado por un vacío; lleno, en el caso de SANAA, del paisaje exterior circundante. $O$ mejor aún: la arquitectura de SANAA se "construye" con materiales como el paisaje y el propio vacío. Porque la equivalencia de todos los elementos es en sí un "invariante", y por tanto, simetría en su obra, precisamente por el equilibrio formado por este juego de "inmaterialidades". Así, los mecanismos de disolución que Mies utilizó en el Pabellón de Barcelona como fueron los reflejos de los materiales satinados, son empleados por SANAA para ir un paso más allá. Es el caso del Pabellón de vidrio del Museo de Arte de Toledo (Ohio, EE.UU.) y los infinitos muros curvos de este material replicándose. ¿̇Cómo expresar sino la condición homogénea e isótropa del espacio? ¿Cómo experimentarlo? Una posibilidad lo dan los espacios no orientados, donde a través de la falta de referencias se pueda traducir, desde el plano perceptivo, como isotropía. Es el desierto, otro, más complejo en sí, el laberinto ${ }^{[18]}$. El Pabellón de Barcelona tiene mucho del último, pero veladamente, deja entrever el primero (Figura 8).

\section{ELIMINAR EL CENTRO}

La intervención que realizó SANAA ${ }^{[19]}$ se enmarca dentro del programa, que en los últimos años se ha llevado a cabo en el Pabellón de Barcelona (FIGURA 9), de instalaciones concebidas como exposiciones ligeras de corta duración que proporcionan la oportunidad de trabajar con formatos creativos diversos. Es sorprendente comprobar, que algunas de las intervenciones que se han dado, han incidido, incrementando o anulando, situaciones de equilibrio de los elementos en clave de simetría. Así, Spectral diffractions de Edwin van der Heide, jugando con el carácter reverberante de los materiales del Pabellón, plantean jerarquías de sonidos desde la emisión independiente de frecuencias parciales y su reflexión. $\bigcirc$ experiencias sobre la ingravidez de Miralles y Tagliabue que acentúan su isotropía, o la anulación de los reflejos de los dos estanques del Pabellón que eliminan la simetría horizontal característica del "ready-made" de Ai Weiwei.

La intervención de SANAA (FIGURA 10) consistió en la instalación de unas cortinas acrílicas transparentes colocadas en forma de espiral en el interior del espacio conocido como la "sala del trono" previsto en la Exposición Universal como escenario para la firma en el libro de visitas por parte de los Reyes de España el día de su inauguración. Como explican ellos mismos:

"Decidimos utilizar un material acrílico para fabricar unos cerramientos transparentes. Imaginamos una instalación que dejaba el espacio del Pabellón de Barcelona intacto. Los cerramientos acrílicos se levantan libremente del suelo formando una espiral silenciosa. El cerramiento rodeaba suavemente el espacio del pabellón y creaba una nueva atmósfera. La visión a través del acrílico cambiaba el original con suaves reflejos que distorsionaban ligeramente el pabellón" (SANAA, 2010, p. 8).

SANAA intensifica el "juego de reflejos" al aumentar tanto las propiedades reflectantes de los propios materiales del Pabellón como los provocados por el propio muro curvo acrílico. Cuando se presentan superficies onduladas dichos reflejos se multiplican de una manera progresiva, a tenor de la propia continuidad de la forma. En este caso una espiral. Realizando

[17] Javier Maderuelo, La idea de espacio en la arquitectura y el arte contemporáneos, 1960-1989 (Madrid: Akal, 2008), p. 16. "el término, confín o lindero de reinos, provincias o posesiones".

[18] En el cuento del AlePH "Los dos reyes y los dos laberintos", para Borges el mayor laberinto es el desierto.

[19] Estuvo expuesta del 20 de noviembre del 2008 al 18 de enero de 2009. Solo dos meses. 
9. Planta del Pabellón de Barcelona según Hilberseimer. Fuente: HILBERSEIMER, Ludwig; Mies van der Rohe, Turín: Città Studi Edizioni, 1993. $1^{a}$ Edición, 1956. 165 pp. ISBN: 978-88-251-0076-1, p. 141. № de fotografía: 144. Pabellón de Alemania de la Exposición Internacional de Barcelona, 1929. 144. Planta.

10. Intervención de SANAA en el Pabellón de Barcelona. Fragmento de la planta de la propuesta definitiva. Fuente: SANAA. Intervention in the Mies van der Rohe Pavilion, Barcelona, Fundació Mies van der Rohe, 2010. ISBN: 978-84-92861187, p. 13. Figura Option C (realized).

11. "Sala del trono" del Pabellón de Barcelona. Fragmento de la planta del Pabellón. Fuente: Peter Carter, Mies at work. Nueva York, Phaidon Press Limited, 2008,71 pp. ISBN: 978-0-7148-9853-7, p. 19. Figura 29.

9
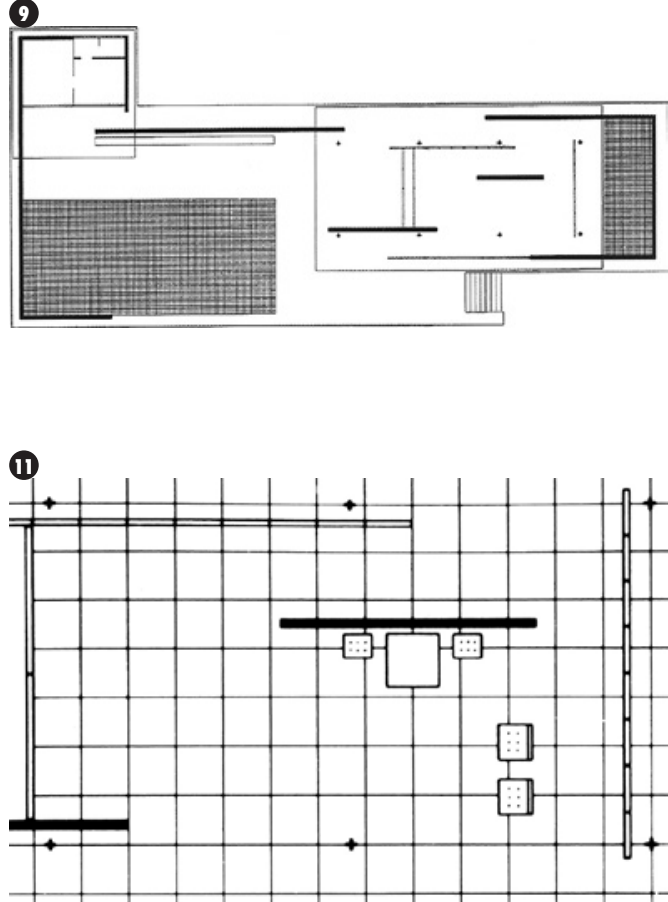

un gesto. Como cuando queremos señalar algo remarcándolo. Pero en este caso consiguiendo lo contrario: disolviendo el centro. El único que existía (FIgura 11). "Las figuras que el ónice producía, su colorido brillante y difuso y sus grandes dimensiones $(235 \times 135 \times 3 \mathrm{~cm}$ cada losa) convertían este rico material en una verdadera joya que provocaba, tal vez con mayor fuerza que cualquier escultura, un centro de interés en el fluido circular por el interior del edificio". Hay algo de premonitorio al utilizar un muro para movilizar una nueva relación entre estructura y espacio ${ }^{[20]}$. En ese muro de ónice, la simetría axial del veteado del mármol señala el lugar de la firma que es distorsionada por la nube de reflejos del acrílico. Conclusión: un pabellón más homogéneo en su condición laberíntica. Un laberinto dentro de otro laberinto, que siguiendo la lógica borgiana es un infinito dentro de otro infinito,

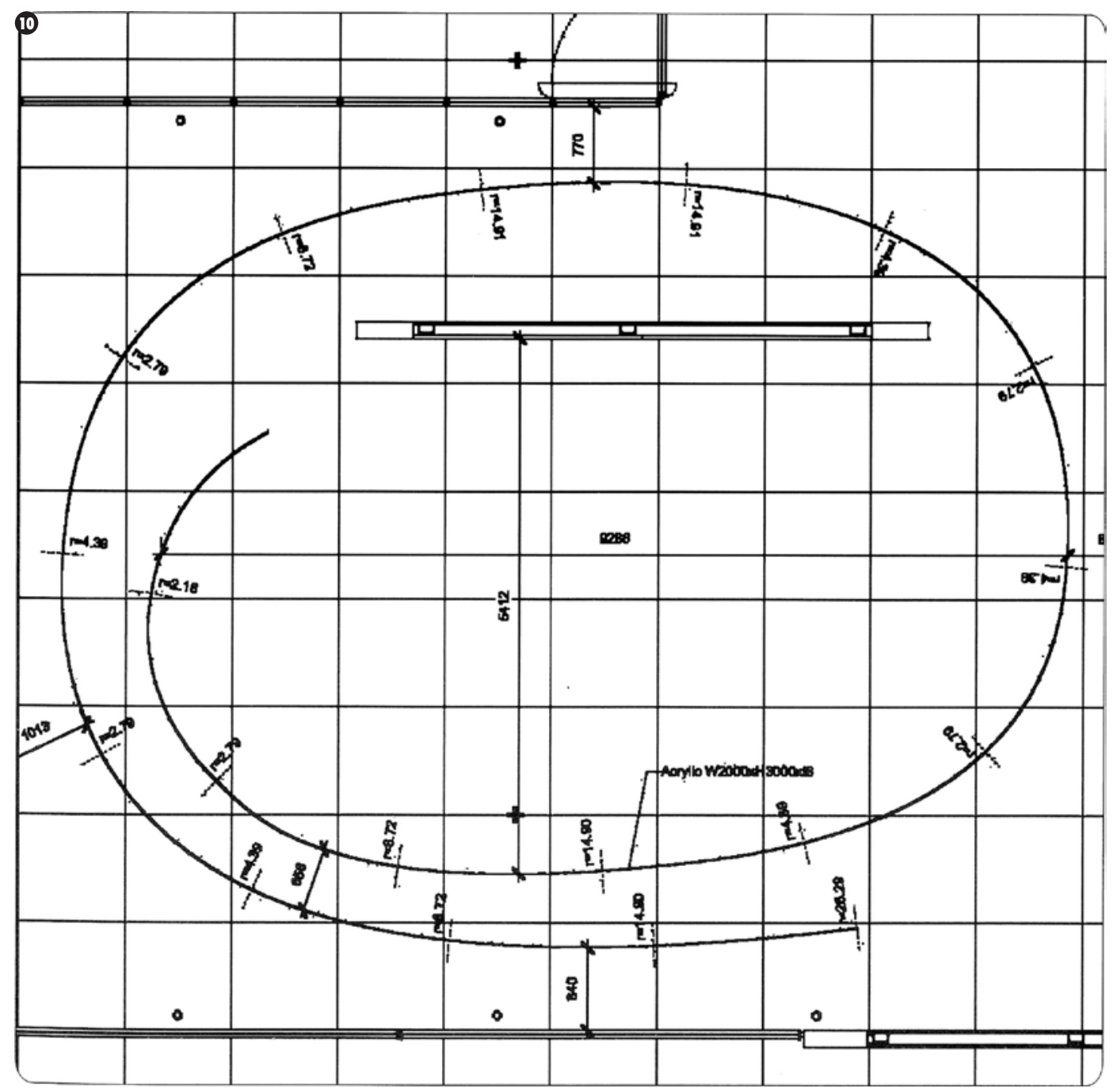

donde los límites aparecen y desaparecen. Se suman o se anulan. Donde no existe la clausura visual, solo distorsionada. La multitud de reflejos que se muestran disuelven el espacio en una atmósfera evanescente, isótropa en su infinitud (FIgURA 12). Es una espiral paradójica. Si no fuese transparente se potenciaría el centro donde se inicia (o en este caso donde se acaba). Al ser su opuesto, lo anula. Un interior-exterior en la intercambiabilidad equivalente entre ambos. Simetría de opuestos que conservan sus propiedades, sin fundirse en un solo espacio. Hay una alternancia de concavidad y convexidad. Pero además es algo más: es la materialización de un recorrido, la geometrización de una trayectoria que elimina la frontalidad que el muro de ónice tenía como protagonista y que obliga a recorrerlo por detrás, a mirarlo, ya desde dentro, en una posición de escorzo. Es una inmersión en un silencio que dilata el espacio. Que lo detiene ingrávido, sin referencias. Una sección 
12. Vista de la intervención de SANAA en el Pabellón. Autor: Ramón Prat @ C. Fundació Mies van der Rohe. http://miesben.com/wp-content/uploads/2014/05/ramon-prat_04-888x382.jpg

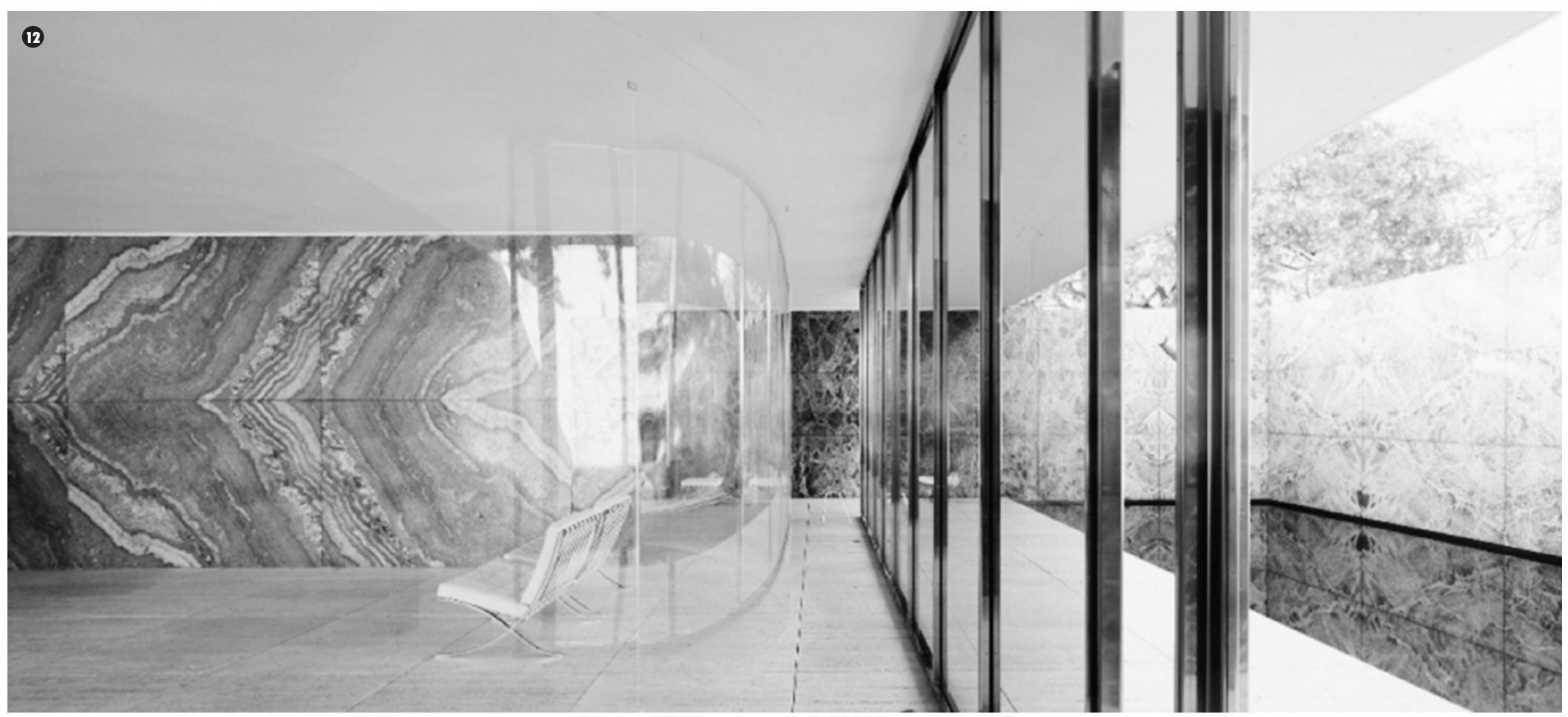

tridimensional invisible que no impide la visión. Solo la deforma. Un manifiesto del pensamiento arquitectónico contemporáneo. Una ofrenda en lo que fue y es el "templo" [21] de la arquitectura moderna.

\section{ESPIRAL TOPOLÓGICA}

Y todo esto, SANAA lo expresa en este trazo construido. En esta espiral silenciosa como un sistema "abierto, ilimitado y sin fronteras concretas entre figura y fondo" (Ruiz de la Puerta, 2007, p. 96). Aquí se encuentra alterada en su forma para poder abrazar al muro de Ónice y al pilar que ocupan la sala del trono. Una espiral que tiene como características intrínsecas la continuidad y la repetición como modelo de crecimiento. En este caso, existe continuidad pero la repetición congruente que configura "un crecimiento asimétrico terminal sin modificación de la forma de la figura" (Thompson, 1980, p. 167) ha desaparecido. Estamos hablando de otro espacio, de otra geometría. Para SANAA esta es una espiral topológica, regular puesto que es "un contorno ondulante topológicamente equivalente a una figura elemental" (Cortes, 2008, p. 38).

Es la esencialidad manifestada en un esquema en planta (FIGURA 13) que en realidad constituye un plano de obra total. Diagrama y construcción en una posición de igualdad. Si lo comparamos con la planta del Pabellón conocida, el código de representación se invierte. El muro curvo equivale ahora al muro de piedra, no al plano de vidrio. Esta materialización "inmaterial" es la prueba de que estamos ante un espacio topológico donde las leyes de la tectónica ya no imponen su lógica: espesores gruesos son los elementos portantes, espesores esbeltos no son estructurales. Aquí es todo lo contrario ${ }^{[22]}$. El muro acrílico curvo "absorbe" la materialidad circundante. Ya antes Mies enseñó el camino. En los aplacados de mármol del Pabellón los veteados e irregularidades confieren a los muros una condición de corte aumentado de materia. Una alteración de escala que nos sitúa junto al bloque extraído de la cantera (FIGURA 14) al que le fue rebanado una laja y que manifiesta en toda su crudeza el interior de la roca que lo constituye. Pero esta impresión queda en entredicho cuando se observa el espesor de los muros. Este desfase es similar al indicado por

21] Quetglas habla de esa condición de templo en el Horror cristalizado.

[22] De hecho, SANAA utiliza estos muros curvos acrílicos como portantes en varias de sus obras como el Museo de Arte de Toledo en Ohio. 
13. Planta intervención de SANAA en el Pabellón de Barcelona. Fragmento del cartel anunciador de la Instalación de SANAA publicado por la Fundación Mies van der Rohe. Arxiu Fundació Mies van der Rohe. Barcelona.

14. Bloque de Onix Doré, procedente de Algeria, antes de empezar el corte, 1986. Autor: Francesc Català-Roca @ Fundació Mies van der Rohe y Fundació Francesc Català-Roca.

$+$

$+$

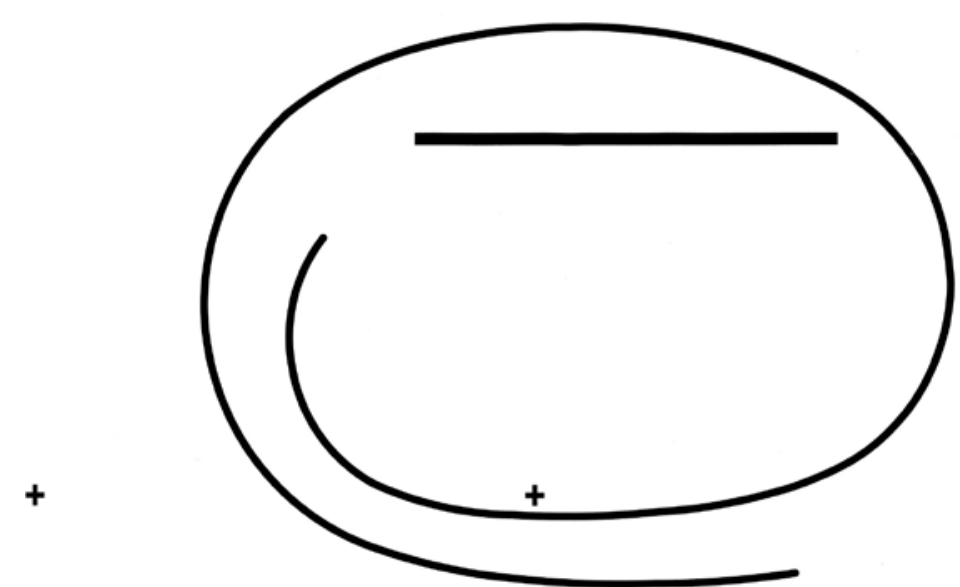

$+$

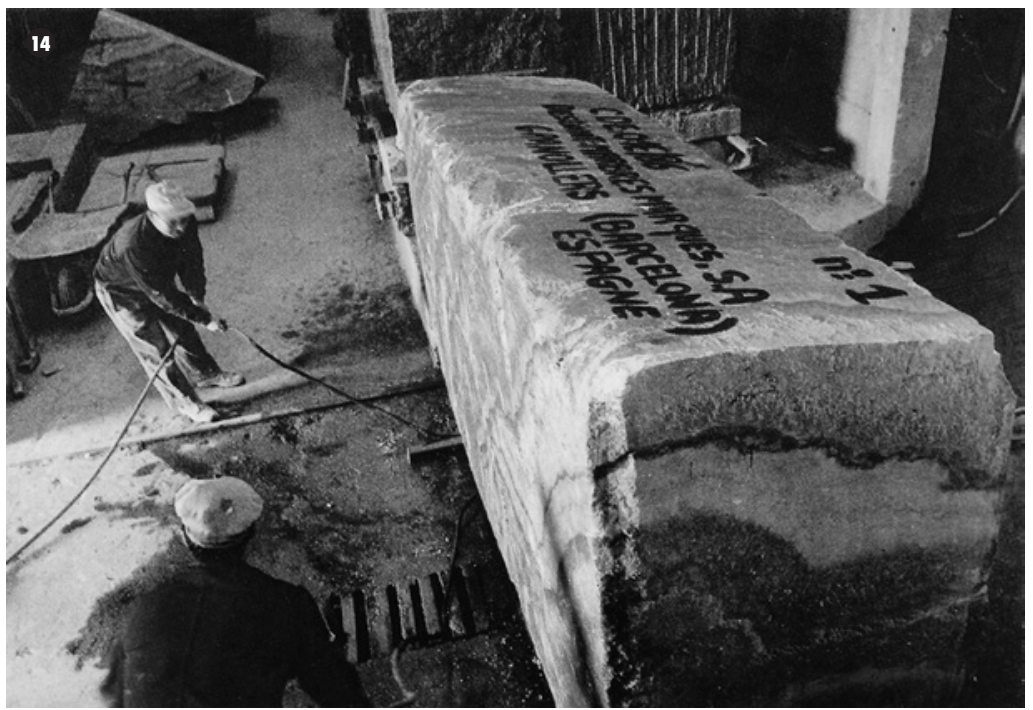

SANAA: la indiferencia entre espesores hasta reducirlo a una pantalla. Pantalla en su doble sentido: expresar un material como una imagen de él y reflejar un universo especular devolviendo el espesor perdido en una naturaleza opuesta: la irreal (FIGURA 15).

\section{(IN)CONCLUSIÓN}

En el único cuaderno de notas que se conserva de Mies van der Rohe, el arquitecto anotó lo siguiente en su hoja tercera: "sobre la técnica y arte del espacio, arte de construir; vale lo mismo" (Neumeyer, p. 410). Como quizás vale lo mismo lo contrario: sobre la técnica y arte de construir, el arte del espacio es equivalente. Después de lo dicho hasta el momento queda el convencimiento, de que la simetría, Mies, SANAA, el Pabellón de Barcelona e incluso el propio espacio no son más que excusas para hablar de lo que es importante en todos ellos: una nueva forma de "hacer arquitectura". E incluso esta, como término huidizo comienza a escaparse de los dedos quedando la sensación incómoda de estar hablando de nada. Y seguramente por esto último, en su imposibilidad indecible, lo escrito tenga algún de valor. Valor por lo indeterminado, lo abierto, lo incompleto. De ahí la (in)conclusión. Una (in)conclusión de pensar que hacer "vibrar unos materiales desperdigados en ese vacío", como fue esa experiencia vivida a finales de los años veinte del siglo pasado, sea la cualidad diferencial "de lo moderno" en la arquitectura de Mies que se transfiere a SANAA. Esa materia "vibrante", en movimiento, que se espacia en su dilatación. Un vacío delimitado, que en el equilibrio de ese acto mantiene esa potencialidad como simetría continua, y se materializa en múltiples espacios. Despejar la paradoja en la similitud. La arquitectura de Mies fue inmaterial siendo extraordinariamente material. Porque lo inmaterial tiene que ver con el "reflejo" de lo material, al cual acoge como modulación de su misma naturaleza. La arquitectura de SANAA es material siendo extraordinariamente inmaterial, porque estamos hablando del espacio como vacío que es simétrico a sí mismo. Es la conexión MiesSANAA proyectada en el tiempo, equivalente e idéntica, análoga en su relación.

\section{REFERENCIAS}

Cortes, J. A. (2008). Topología arquitectónica. Una indagación sobre la naturaleza del espacio contemporáneo. Croquis, № 138 SANAA [Kazuyo Sejima + Ryue Nishizawa]. http://doi.org/02125633

Corrales, Capi (2002) Contando el espacio (Despacio Ediciones). Madrid.

De Solá Morales, I., Cirici, C., \& Ramos, F. (1993). Mies van der Rohe: El Pabellón de Barcelona. (G. Gili, Ed.). Barcelona.

Evans, R. (2005). Las simetrías paradójicas de Mies van der Rohe. In Traducciones (p. 312). Gerona: Pre-textos.

Jaraiz, J. (2013). SANAA: espacios, límites y jerarquías. Buenos Aires: Diseño.

Koolhaas, R. (2004). Miestakes. A+t.

Lederman, L. M., \& Hill, T. C. (2006). La simetría y la belleza del universo. Barcelona: Tusquets.

Martínez Santa-María, Luis (2004) El árbol, el camino, el estanque, ante la casa. Barcelona: ArquiaTesis.

Navarro Baldeweg, J. (1999). La habitación vacante (p. 176). Gerona: Pre-textos 
15. Detalle del muro de Onix Doré. Autor: Foto propia. Francisco Muñoz Carabias. Tomada con una PENTAX K7. Agosto del 2013. Autorización Fundació Mies van der Rohe. Barcelona.
Neumeyer, Fritz, (2009) Mies van der Rohe: la palabra sin artificio. Reflexiones sobre arquitectura 1922 1968 Madrid: El Croquis. (Mies van der Rohe; Cuaderno de notas, 1928).

Quetglas, J. (2001). El horror cristalizado. Imágenes del Pabellón de Alemania de Mies van der Rohe. Barcelona: Actar.

Rowe, C. (1999). Manierismo y arquitectura moderna y otros ensayos ( $3^{a}$ ed.). Barcelona: Gustavo Gili. Ruiz de la Puerta, F. (2007). La espiral en la arquitectura. Madrid: Mairea.

SANAA. (2010). Intervención en el Pabellón de Mies van der Rohe. (Actar, Ed.). Barcelona.

Schulze, F. (1986). Mies van der Rohe. Una autobiografía crítica. Madrid: Hermann Blume.

Thompson, D. (1980). Sobre el crecimiento y la forma. Madrid: Blume.

Weyl, H. (1990). Simetría. Madrid: McGraw-Hill.

Ynzenga Acha, B. (2013). La materia del espacio arquitectónico. Buenos Aires: Nobuko.

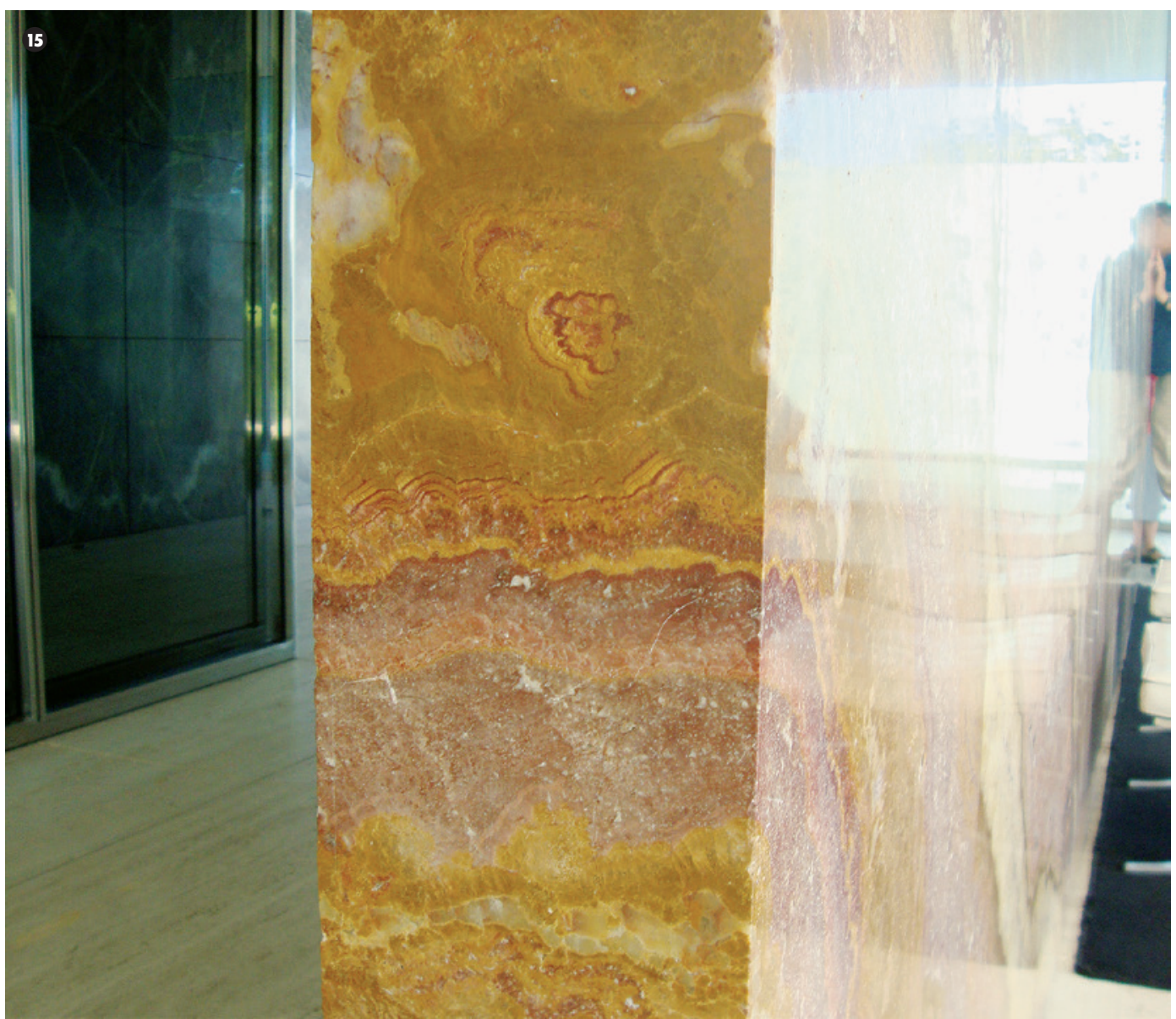

